\title{
Dexmedetomidine-induced contraction in the human umbilical artery
}

\author{
Lee $\mathrm{SH}^{1}$, Sohn $\mathrm{JT}^{1,2}$ \\ Department of Anesthesiology and Pain Medicine, Gyeongsang National University College of Medicine, Gyeongsang National \\ University Hospital, Gyeongsang, Republic of Korea. jtsohn@gnu.ac.kr
}

Text in PDF www.elis.sk.

\section{Dear Editor}

I have read your interesting article entitled, "In vitro vasoactive effects of dexmedetomidine on isolated human umbilical arteries," recently published in Bratisl Med J (1). The alpha-2 adrenoceptor agonist dexmedetomidine is widely used for sedation (2). The following comments should be considered to understand this article (1). First, two cumulative dexmedetomidine dose-response curves were generated in the same human umbilical artery (HUA) before and after treatment with inhibitors. Dexmedetomidine $\left(2 \times 10^{-5}\right.$ $\mathrm{M})$-induced maximal contraction was used as the reference value of contraction evoked by dexmedetomidine (1). However, it is important to confirm HUA viability by assessing contractility to $\mathrm{KCl}$ or 5-hydroxytryptamine and the value generated can be used as a reference instead of that generated by dexmedetomidine $\left(2 \times 10^{-5} \mathrm{M}\right)$ in the present experiment $(1,3)$. Additionally, as the HUA strip produces spontaneous contraction and shows wide variability in vasoreactivity to a vasoconstrictor and inconsistency of vasoreactivity over time in the same HUA, KCl-induced contraction contributes to decreased spontaneous contraction and reduced variability of vasoreactivity and may stabilize the HUA $(3,4)$. Furthermore, two dexmedetomidine dose-response curves generated in the same HUA even without inhibitors may not be identical (1). Thus, a comparison should be performed. It is not clear how it can be verified that dexmedetomidine used in the first dexmedetomidine dose-response curve is completely washed out before the second experiment to generate a dose-response curve in the presence of the inhibitors. Second, the clinically relevant concentration of dexmedetomidine used for sedation in humans approximately corresponds to $10^{-8} \mathrm{M}$. Third, prazosin $\left(3 \times 10^{-9} \mathrm{M}\right)$, an alpha- 1 adrenoceptor inhibitor, inhibits dexmedetomidine-induced contraction in isolated rat aorta, whereas a very high concentra-

Department of Anesthesiology and Pain Medicine, Gyeongsang National University College of Medicine, Gyeongsang National University Hospital, 15 Jinju-daero 816 beon-gil, Jinju-si, Gyeongsangnam-do 52727, Republic of Korea Institute of Health Sciences, Gyeongsang National University, Jinju-si, 52727, Republic of Korea

Address for correspondence: JT Sohn, Department of Anesthesiology and Pain Medicine, Gyeongsang National University Hospital, 79 Gangnam-ro, Jinju-si, 52727, Republic of Korea.

Phone: +82.55.7508586, Fax: +82.55.7508142 tion of prazosin $\left(10^{-5} \mathrm{M}\right)$ attenuated vasoconstriction induced by all concentrations of dexmedetomidine in the $\operatorname{HUA}(1,5)$. This prazosin-mediated inhibition of dexmedetomidine-induced contraction may be associated with non-specific actions. Fourth, as cumulative dexmedetomidine dose-response curves were evaluated in the reported study, using a line graph appropriate for a cumulative dose-response curve instead of a bar graph in Figures 2 and 3 would be more helpful for readers to understand Figures 1 and 2 (1). In addition, for the comparison of the magnitude of cumulative dexmedetomidine-induced contraction in the presence or absence of inhibitors in the same HUA, it is more reasonable to use a linear mixed effect model or two-way repeated measure analysis of variance followed by Bonferroni's multiple comparison test rather than Wilcoxon rank test, which was used in the reported study (1). p value used in the Wilcoxon rank test in the reported study should be adjusted to about $0.008(p=0.05 / 6)$ to reduce the type 1 error (1). I believe that this study contributes to understanding the mechanism associated with dexmedetomidineinduced contraction in HUA.

\section{Reference}

1. Arun O, Taylan SB, Duman I et al. In vitro vasoactive effects of dexmedetomidine on isolated human umbilical arteries. Bratisl Med J 2019; 120 (1): 40-45.

2. Mathur PR, Jain N, Kumar A, Thada B, Mathur V, Garg D. Comparison between lignocaine nebulization and airway nerve block for awake fiberoptic bronchoscopy-guided nasotracheal intubation: a single-blind randomized prospective study. Korean J Anesthesiol 2018; 71 (2): 120-126.

3. Monuszko E, Halevy S, Freese K, Liu-Barnett M, Altura B. Vasoactive actions of local anaesthetics on human isolated umbilical veins and arteries. Br J Pharmacol 1989; 97 (2): 319-328.

4. Altura BM, Malaviya D, Reich CF, Orkin LR. Effects of vasoactive agents on isolated human umbilical arteries and veins. Am J Physiol 1972; 222 (2): 345-355.

5. Byon HJ, Ok SH, Lee SH, Kang S, Cho Y, Han JY, Sohn JT. Dexmedetomidine inhibits phenylephrine-induced contractions via alpha-1 adrenoceptor blockade and nitric oxide release in isolated rat aortae. Int J Med Sci 2017; 14 (2): 143-149.

Received February 4, 2019. Accepted February 12, 2019. 